\title{
Dilaceração radicular: análise radiográfica em pacientes com fissura labial e/ou palatina não sindrômica
}

\author{
Root Dilaceration: Radiographic Analysis in Nonsyndromic \\ Cleft Lip with or without Cleft Palate (NSCL \pm P) Patients
}

\author{
Laís Bastos Guimarães', Gabriela dos Santos Lopes', Paula Santana Bezerra', \\ Vitória Rodrigues de Santana', Alena Peixoto Medrado², Silvia Regina de Almeida Reis ${ }^{3}$ \\ 'Escola Bahiana de Medicina e Saúde Pública. Salvador, Bahia, Brasil. laisguimaraes.pos@bahiana.edu.br, gabrielalopes.pos@bahiana.edu.br, \\ paulabezerra14.1@bahiana.edu.br, vitoriasantana14.2@bahiana.edu.br \\ ${ }^{2}$ Universidade Federal da Bahia e Escola Bahiana de Medicina e Saúde Pública. Salvador, Bahia, Brasil. apmedrado@bahiana.edu.br \\ ${ }^{3}$ Autora para correspondência. Escola Bahiana de Medicina e Saúde Pública. Salvador, Bahia, Brasil. srareis@bahiana.edu.br
}

\begin{abstract}
Resumo | Fissuras labiais e/ou palatinas não sindrômicas (FL/PNS) representam a anomalia congênita mais comum em humanos. Entretanto, são poucos os estudos que analisam a curvatura e dilaceração em pacientes acometidos pela FL/PNS. O objetivo deste estudo foi avaliar a prevalência de dilaceração radicular em 400 indivíduos portadores de $\mathrm{FL} / \mathrm{PNS}$, assim como relacionar o grau de curvatura da raiz com os diferentes tipos de fissuras. Observou-se que raízes de pré-molares e molares apresentaram diferentes direções $(p<0,001)$. Raízes retas e inclinadas para a face distal foram mais prevalentes $(46,6 \%$ e $44,5 \%$ respectivamente). Existem diferenças quanto a localização da curvatura em relação ao $1 / 3$ radicular $(p<0,001)$. As fissuras labiais e as fissuras palatinas apresentaram maior frequência de dilaceração radicular. Não foi observado associação significativa do grau de curvatura radicular dos molares e pré-molares a um tipo específico de fissura. Esses resultados sugerem a realização de estudo caso-controle para que questões relativas às diferenças de angulação entre os portadores de $\mathrm{FL} / \mathrm{PNS}$ e a população em geral, sejam respondidas.
\end{abstract}

\begin{abstract}
Nonsyndromic cleft lip with or without cleft palate (NSCL $\pm P$ ) represents the most common congenital abnormality in humans. Nevertheless, there are few studies that analyze the curvature and dilaceration in NSCL $\pm P$ patients. The purpose of this study was to evaluate the prevalence of root dilaceration in $400 \mathrm{NSCL} \pm \mathrm{P}$ patients, as well as relate the root curvature degree with different kinds of clefts. It was observed that premolars and molars roots have different directions $(p<0.001)$. Straight roots inclined to the distal face were more prevalent $(46.6 \%$ and $44.5 \%$ respectively). There are differences in the location of the curvature in relation to the $1 / 3$ root $(p$ $<0.001)$. The cleft lip only and the cleft palate only had presented a higher frequency of root dilaceration. There was no significant association between the degree of root curvature of molars and premolars to a specific kind of cleft. These findings suggest to conduct a case-control study to answer questions relating to differences in angulation among NSCL $\pm P$ patients with the general population.
\end{abstract}

Keywords: Cleft lip, cleft palate, dental abnormalities.

Palavras-chave: Fissura labial, fenda palatina, anormalidades dentárias. 


\section{Introdução}

Fissura de lábio e/ou palato não sindrômica (FL/ PNS) compreende a malformação mais comum da região craniofacial. A maioria dos indivíduos portadores dessas anomalias apresentam a forma não sindrômica (FL/PNS), cujas fendas não estão associadas a outras malformações e nem a alterações comportamentais ou cognitivas'. Entretanto, mais de 400 síndromes que apresentam a $\mathrm{FL} / \mathrm{P}$ no aspecto clínico já foram descritas na literatura².

A prevalência mundial de $\mathrm{FL} / \mathrm{PNS}$ é de aproximadamente 1 em cada 500 nascidos vivos e varia de acordo com a localização geográfica e etnia/raça ${ }^{3}$. Os pacientes afetados por esta malformação congênita apresentam efeitos sobre a fala, audição, aparência e cognição, o que acarreta consequências tanto para a saúde quanto para a integração social do indivíduo ${ }^{4}$. Do ponto de vista embriológico, as $\mathrm{FL} / \mathrm{PNS}$ são resultantes de defeitos na fusão dos processos craniofaciais que formam $\circ$ palato primário e secundário, no primeiro trimestre do desenvolvimento intra-uterino ${ }^{3}$. Sua etiopatogenia ainda é pouco compreendida, entretanto há um consenso de que se trata de uma condição multifatorial, que pode ser determinada pela interação de diferentes lócus gênicos com co-variáveis ambientais adicionais. Fatores ambientais como o tabagismo, álcool, exposição a medicamentos e má nutrição associados a heterogeneidade genética pode aumentar 0 risco no desenvolvimento da FL/PNS durante o período gestacional ${ }^{5}$.

De acordo com as características clínicas, as fissuras podem ser classificadas em três grupos principais, tendo como referência anatômica o forame incisivo, em: (1) fissuras pré- forame incisivo ou fissuras labiais (FL); (2) fissuras pós-forame incisivo ou fissuras palatinas (FP); (3) fissuras transforame incisivo ou fissuras lábio- palatinas (FLP) ${ }^{6}$.

Estudos em diversas populações demonstraram que os indivíduos com FL/PNS apresentam alterações dentárias nas regiões das fissuras, entre incisivos e caninos, como também fora desta região, especial- mente em pré-molares e molares. As mais comuns são as alterações de número, tamanho e posição $0^{7,8,9}$. Sabe-se que a embriologia dentária está estritamente associada ao desenvolvimento craniofacial. Pesquisas demonstram que a odontogênese, a formação do lábio e do palato apresentam semelhanças embriológicas e ocorrem em período espacial e temporal semelhantes, além da participação de muitos genes em comum ${ }^{8,10}$. Assim, salienta-se que a presença de anomalias dentárias em pacientes fissurados possa indicar uma origem genética em comum entre estas alterações ${ }^{11}$.

Dentre as anomalias pouco estudadas nos fissurados, encontra-se a dilaceração radicular. Esta malformação é definida como uma angulação na raiz do dente. Segundo Colack et.al ${ }^{12}$ e Malcic et.al ${ }^{13}$, a dilaceração é considerada apenas quando o ângulo de curvatura radicular é maior ou igual a 900. Já Chohayeb et.al ${ }^{14}$, atribuem a dilaceração quando o ângulo de uma raiz dentária é igual ou maior que 200. Os estudos na literatura sobre a dilaceração radicular ainda são controversos, em especial aqueles relacionados aos portadores de FL/PNS.

Apesar da dilaceração não constituir qualquer comprometimento vital à saúde do indivíduo, pode acarretar complicações durante o tratamento endodôntico ou cirúrgico, quando não diagnosticada. Acredita-se que a análise da curvatura das raízes é fundamental para um tratamento odontológico planejado e mais eficaz. Neste contexto, essa pesquisa tem como objetivo avaliar a prevalência de dilaceração radicular em indivíduos portadores de $\mathrm{FL} /$ PNS, assim como relacionar o grau de curvatura da raiz com os diferentes tipos de fissuras.

\section{Metodologia}

\section{Aprovação do comitê de ética em pesquisa}

O presente estudo foi realizado de acordo com as normas relativas à ética em pesquisa envolvendo seres humanos. Foi obtida deliberação do Comitê 
de Ética em Pesquisa do Hospital Santo Antônio, protocolo número 48777315.0.0000.0047.

\section{População}

Este estudo transversal, retrospectivo foi constituído por uma amostra de 400 pacientes portadores de fissura labial (FL), fissura labiopalatina (FLP) e fissura palatina (FP) não sindrômicas, que possuíam radiografia panorâmica e histórico odontológico completo. Esses indivíduos foram provenientes do Centro de Fissuras Orofaciais do Hospital Santo Antônio, em Salvador-Bahia. Pacientes com idade inferior a 12 anos e aqueles portadores de anomalias congênitas e funcionais não foram incluídos na amostra. Para aqueles que já haviam sido submetidos ao tratamento ortodôntico ou que ainda estavam em tratamento regular, a primeira radiografia panorâmica de diagnóstico foi analisada.

Os dados de cada paciente coletados do prontuário da instituição foram anotados em ficha padronizada com identificação, gênero, idade no momento da radiografia e tipo de fissura. Foram também anotados os fenótipos quanto à extensão, se completa ou incompleta e quanto à localização da malformação, se unilateral ou bilateral.

\section{Análise imaginológica}

As radiografias com níveis aceitáveis de nitidez, contraste e densidade foram, primeiramente, examinadas através do negatoscópio de luz homogênea e difusa, com auxílio da lupa com 4 vezes de aumento, em uma sala escura para a identificação inicial da curvatura das raízes. Foi realizada blindagem das margens entre a radiografia e o negatoscópio com papel de cartolina preto. Posteriormente, as radiografias foram fotografadas através de uma máquina digital (Canon T2i) com lente macro 100x, cujas imagens foram capturadas mantendo resolução fixa de $300 \mathrm{dpi}$, arquivadas em computador e salvas no formato JPEG. Foi utilizado o software de análise de imagens OsiriX Lite, Source Forge, Los Angeles, Califórnia, USA. Este software está disponibilizado gratuitamente no site www.osirix-viewer.com/Down- loads.html. Para melhorar a acuidade visual do ápice dentário, foi utilizado o recurso imaginológico de inversão de filtro (filtro negativo) com zoom de $4 \mathrm{x}$ pelo software Adobe Photoshop 5.1 (Adobe Systems Inc., San Jose, CA, USA).

Os dentes analisados foram os pré-molares e molares inferiores. Não foram inclú́dos no estudo a dentição decídua, terceiros molares, dentes com patologias que afetavam a raiz, dentes com restaurações MOD, dentes com formação incompleta da raiz, com destruição coronária, alterações na câmara pulpar que comprometia a visibilidade da junção amelocementária e radiografias que apresentavam menos de um pré-molar e molar de cada lado. A curvatura das raízes foi classificada de acordo com Fuentes et.al $\left.\right|^{15}$ em quatro grupos: reta $\left(0^{\circ}\right)$, leve $\left(>0^{\circ}\right.$ e $15^{\circ}$ $\leq)$, moderada $\left(>15^{\circ}\right.$ e $\left.\leq 30^{\circ}\right)$ e severa $\left(>30^{\circ}\right)$. Também foi classificada em relação às faces mesial e/ ou distal e de acordo com o terço radicular (apical, médio e coronal). Outros critérios de classificação utilizada para avaliação da dilaceração foi o adotado por Colak et.al ${ }^{12}$; Malcic et.al ${ }^{13}$ e Hamasha et.al ${ }^{16}$ (angulação $\geq 90^{\circ}$ ) e por Chohayeb et.al ${ }^{14}$ (angulação $\geq 20^{\circ}$ ).

Para a determinação do ângulo da curvatura radicular sobre a imagem digitalizada, foi traçada uma linha reta na junção amelocementária unindo as faces mesial e distal do dente, determinada como "Ll". O primeiro ponto a ser identificado foi o ponto "A", que representa o teto da câmara pulpar, de tal forma, que coincide com o centro da coroa dentária. $O$ segundo ponto determinado foi o ponto "B", no centro do canal radicular localizado a 5 $\mathrm{mm}$ em direção apical a "L1". Uma segunda linha perpendicular a "Ll", foi efetuada em relação ao longo eixo do dente seguindo o canal radicular, denominada linha "L2". A última linha (L3) foi feita a partir do centro do forame apical no ponto "C" até - local onde a linha L2 abandona o canal radicular, denominado de ponto " $D$ ". O ângulo formado pela interseção entre as linhas $L 2$ e $L 3$, foi definido como ○ ângulo de curvatura radicular, o ponto " $X$ " (Figura 1). Para a avaliação dos molares, a linha "LI" foi posicionada sobre o assoalho da polpa (Figura 1E). 


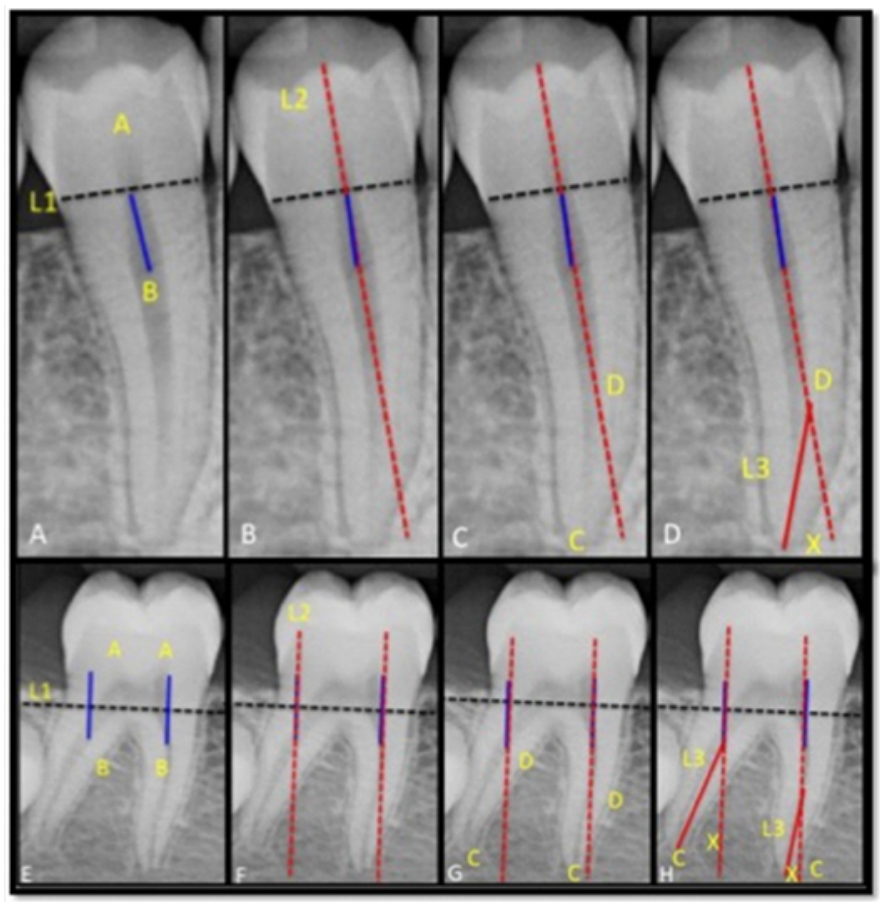

Figura 1. Linhas de referência para o cálculo do ângulo de curvatura radicular no pré-molar e no molar inferior. Linha tracejada em preto indica a junção amelocementária no pré-molar e assoalho da polpa no molar. Interseção entre as linhas vermelhas indica o ângulo de curvatura da raiz, ângulo X. Método de aferição adaptado de Fuentes et al.

Durante a análise das imagens, dois investigadores examinaram separadamente cada radiografia quanto a presença de dilaceração radicular e grau de curvatura das raízes. A variação interexaminador foi calculada comparando-se os resultados dos dois pesquisadores. $O$ examinador 1 obteve índice Kappa de 0,87 e o examinador 2 o índice de 0,97 . Para eliminar a variação intraexaminador, as imagens foram revisadas duas vezes pelo mesmo pesquisador, separadamente, em ocasiões diferentes, com um intervalo de duas semanas. A variação interexaminador foi de 0,80 . Esses resultados sugerem a consistência de cada examinador durante 0 estudo.

\section{Análise estatística}

A associação entre o tipo de fissura e a prevalência das variáveis relativas à curvatura radicular foi avaliada através dos testes Qui-quadrado e exato de Fisher, com nível de significância estatística de $p<0.05$. A regressão multinominal definiu $\circ$ risco atribuído a curvatura radicular $\left(>20^{\circ}\right)$ em relação aos diferentes tipos de fissura por grupo de dentes. A fissura labiopalatina (FLP) foi utilizada como referência para cada uma das variáveis estudadas, com intervalo de confiança (IC) de $95 \%$.

\section{Resultados}

\section{Características da amostra}

Dos 400 indivíduos com FL/PNS analisados neste estudo, $227(56,7 \%)$ pertenciam ao gênero feminino e $173(43,25 \%)$ ao gênero masculino. A média de idade foi de $15+/-7,3$ anos. A FLP foi a malformação mais comum $(59,25 \%)$. A FL foi identificada em $23,5 \%$ da amostra enquanto que a FP correspondeu a $17,25 \%$ dos casos. Em geral as fendas completas foram mais observadas $(n=314)$ do que as incompletas ( $n=86$ ) e as fendas unilaterais foram mais frequentes $(n=301)$ do que as bilaterais $(\mathrm{n}=99)$. Foram analisados 2.456 dentes, e um total de 4.088 raízes.

\section{Distribuição da direção da raiz e grau da curvatura}

A tabela 1 mostra a distribuição da direção da raiz por grupo de dentes. Observou-se diferenças estaticamente significantes na direção das raízes em relação aos pré-molares e molares $(p<0,001)$. Dentes pré-molares e molares apresentaram diferentes direções de curvatura. Raízes retas e inclinadas para a face distal mostraram maiores índices $(46,6 \%$ e $44,5 \%$ respectivamente). 


\begin{tabular}{|c|c|c|c|c|c|c|c|}
\hline \multirow{3}{*}{ Grupo de dentes } & \multicolumn{6}{|c|}{ Direção da raiz } & \multirow{3}{*}{$\mathbf{p}$} \\
\hline & \multicolumn{2}{|c|}{ Reta } & \multicolumn{2}{|c|}{ Mesial } & \multicolumn{2}{|c|}{ Distal } & \\
\hline & $\mathrm{n}$ & $\%$ & $\mathrm{n}$ & $\%$ & $\mathrm{n}$ & $\%$ & \\
\hline & & & & & & & $<0,001$ \\
\hline $1^{\circ}$ pré-molar & 512 & 70,6 & 99 & 13,6 & 115 & 15,8 & \\
\hline $2^{\circ}$ pré-molar & 451 & 64,7 & 34 & 4,9 & 212 & 30,4 & \\
\hline $1^{\circ}$ molar & 321 & 24,5 & 137 & 10,5 & 849 & 65,0 & \\
\hline $2^{\circ}$ molar & 619 & 45,6 & 93 & 6,8 & 646 & 47,6 & \\
\hline Total & 1903 & 46,6 & 363 & 8,9 & 1822 & 44,5 & \\
\hline
\end{tabular}

Observou-se também diferenças quanto a localização da curvatura em relação ao $1 / 3$ radicular $(p<0,001)$. A porção coronal foi a menos acometida $(0,4 \%)$, em relação às demais (apical: $27,7 \%$ e medial: $25,3 \%$ ), tabela 2.

Tabela 2. Distribuição da localização da curvatura em relação ao terço radicular em portadores de FLP/PNS.

\begin{tabular}{|c|c|c|c|c|c|c|c|}
\hline \multirow{3}{*}{ Grupo de dentes } & \multicolumn{6}{|c|}{$1 / 3$ radicular } & \multirow{3}{*}{$\mathbf{p}$} \\
\hline & \multicolumn{2}{|c|}{ Apical } & \multicolumn{2}{|c|}{ Medial } & \multicolumn{2}{|c|}{ Coronal } & \\
\hline & $\mathrm{n}$ & $\%$ & $\mathrm{n}$ & $\%$ & $\mathrm{n}$ & $\%$ & \\
\hline & & & & & & & $<0,001$ \\
\hline $1^{\circ}$ pré-molar & 120 & 16,5 & 93 & 12,8 & 1 & 0,1 & \\
\hline $2^{\circ}$ pré-molar & 142 & 20,4 & 104 & 14,9 & - & - & \\
\hline $1^{\circ}$ molar & 466 & 35,7 & 505 & 38,6 & 12 & 0,9 & \\
\hline $2^{\circ}$ molar & 404 & 29,7 & 334 & 24,4 & 4 & 0,3 & \\
\hline Total & 1132 & 27,7 & 1036 & 25,3 & 17 & 0,4 & \\
\hline
\end{tabular}

O grau de curvatura quando classificado de acordo com Fuentes et.al ${ }^{16}$ e Chohayeb et.al ${ }^{14}$ foi estaticamente significante em relação aos dentes analisados nos diferentes tipos de fissura $(p<0,001)$. Em todos os tipos de fissura, notou-se que a maioria dos portadores apresentou raízes retas e com grau de curvatura moderada, enquanto que os graus leve e severo apresentaram menor frequência. Segundo a classificação Chohayeb et.al ${ }^{14}$, a fissura palatina e a fissura labial foram as que apresentaram maior frequência de angulação $\left(\geq 20^{\circ}\right)$, tabela 3 .

Observou-se que o grau de curvatura radicular de molares e pré-molares não está associado a um tipo específico de fissura, mesmo quando utilizou-se a classificação de Chohayeb et.al ${ }^{14}\left(\geq 20^{\circ}\right)$. Entretanto, um índice limítrofe $(p=0,059)$ foi observado em relação aos molares, tabela 4 .

Na tabela 5 percebe-se que na presença de FP, o risco de dilaceração radicular nos pré-molares esquerdos foi duas vezes maior do que em relação a FLP (FP: RR 2,09; IC 95\% 0,98-4,48, p=0,055). Já nos pré-molares direitos, foi observado diminuição do risco de dilaceração na presença de FP em relação a FLP (FP: RR 0,65; IC 95\% 0,29 - 1,41), porém sem significância estatística $(p=0,271)$. Quando o risco de dilaceração dos molares foi analisado, observou-se valores próximos para a FP e FL em relação a FLP e sem significância estatística. 


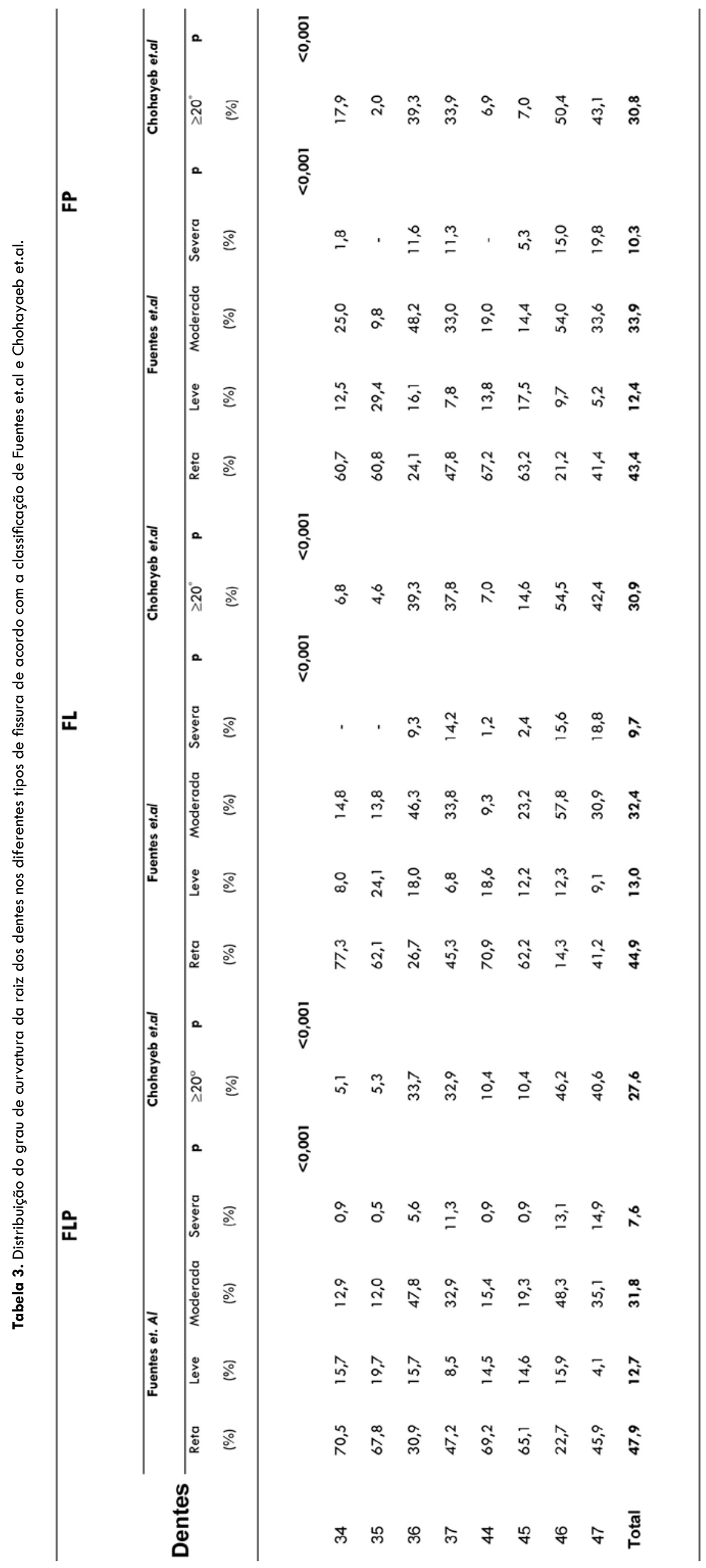




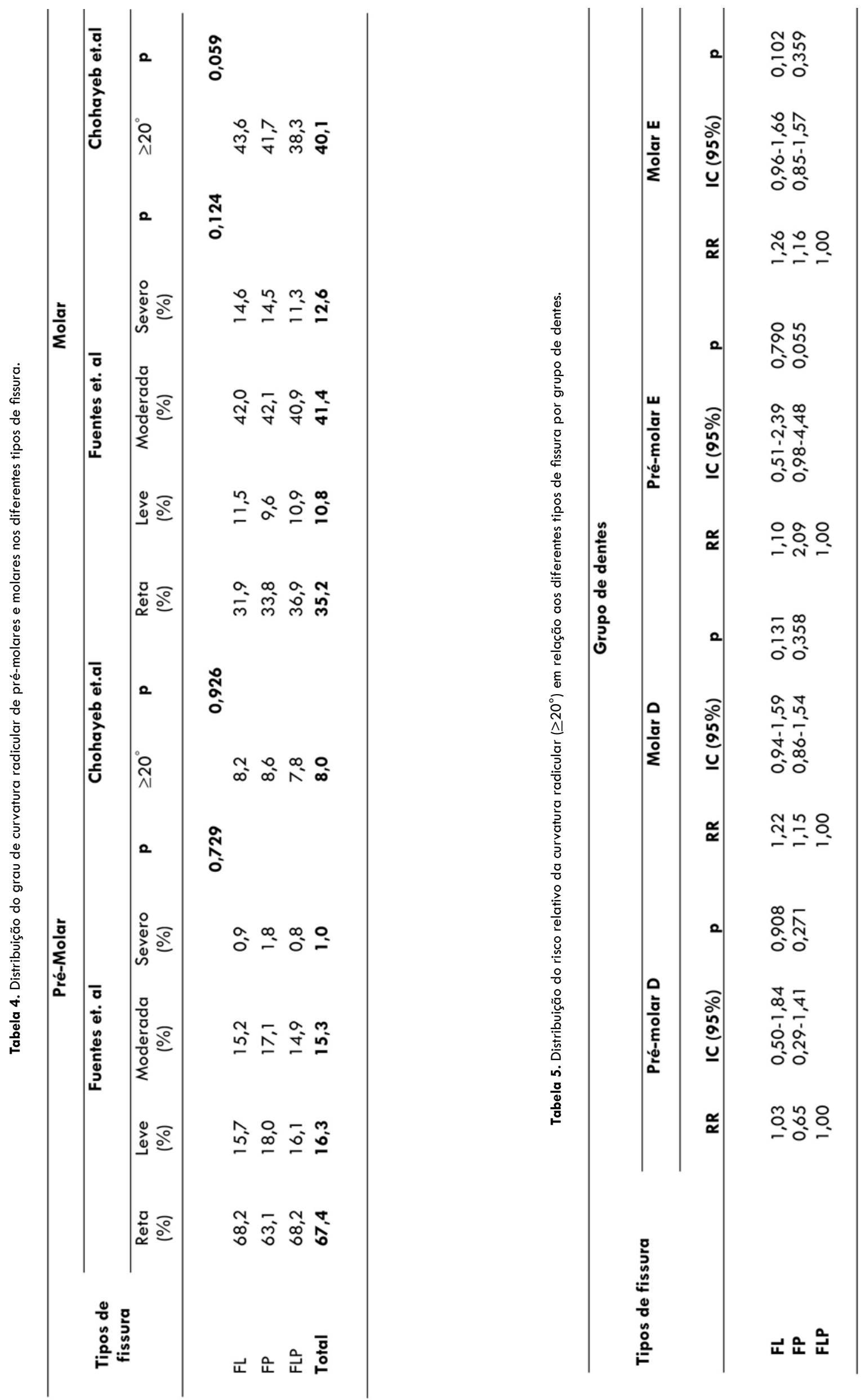




\section{Discussão}

O Centro de Anomalias Craniofaciais das Obras Sociais Irmã Dulce é uma referência no tratamento de indivíduos com fissuras orofaciais não apenas na Bahia, como também nos estados do Norte e Nordeste do Brasil. Atualmente, a entidade filantrópica abriga um dos maiores complexos de saúde do país, com cerca de 4,6 milhões de atendimentos ambulatoriais por ano a usuários do Sistema Único de Saúde. Entretanto, até o presente momento, ainda não existem trabalhos neste Centro sobre a análise da curvatura de pré-molares e molares em pacientes com fissuras labiais e/ou palatinas não sindrômicas.

Na literatura questiona-se o uso de radiografia panorâmica como método de escolha no diagnóstico da dilaceração radicular ${ }^{17}$. Em pesquisas sobre dilaceração, alguns autores ultilizaram a radiografia periapical, sugerindo que este tipo de exame apresenta maior precisão da imagem ${ }^{16,17}$. No presente trabalho, foi utilizada radiografia panorâmica por se tratar de um estudo retrospectivo, cuja radiografia e informações foram retiradas de prontuários dos portadores de FL/PNS no inicio do tratamento. A radiografia panorâmica foi o primeiro exame solicitado para auxílio no diagnóstico dos pacientes atendidos. Nesse tipo de exame, os pré-molares e molares inferiores são bem visualizados, com pouca distorção, daí a escolha em estudar esses elementos dentários. Weckwert et.al $2016^{18}$ também analisaram a dilaceração radicular em indivíduos com fissuras labiopalatinas através de radiografias panorâmicas e salientaram que este tipo de exame é uma boa opção na visualização da curvatura radicular.

Segundo Colak et.al ${ }^{12}$ as raízes dos dentes apresentam um grau normal de curvatura de acordo com a sua localização anatômica. Classificar o grau de curvatura das raízes é importante, pois casos de curvaturas excessivas são considerados dilaceração radicular e influenciam no eventual tratamento dessas unidades dentárias.

A dilaceração radicular pode ocorrer devido alguns fatores como consequência de um trauma mecânico na porção calcificada de um dente predecessor primário, fenda labial ${ }^{19}$, desenvolvimento de um germe dentário ectópico ${ }^{20}$ e fatores hereditários ${ }^{21}$.
Na literatura existem várias definições sobre a dilaceração radicular, porém não há um consenso quanto a sua classificação. A dilaceração é considerada quando há uma curvatura anormal da raiz22, que pode ser a partir de um ângulo $\geq 90^{\circ} 12,13,16,18$ ou $\geq 20^{\circ 14}$. Os autores em sua maioria não descrevem um método para o diagnóstico da dilaceração radicular, apenas classificam quando um elemento dental apresenta uma curvatura acentuada em relação ao seu longo eixo. A maioria dos estudos, porém, não realizam a mensuração das raízes, fazem apenas por inspeção visual ${ }^{23,24}$. Estas discrepâncias no método de diagnóstico e os diferentes critérios utilizados, torna difícil uma real definição para esta anomalia dentária.

Na presente pesquisa não foi verificado angulação $>90^{\circ}$ em nenhuma das raízes analisadas, independente se de pré-molares ou molares. Esses resultados são consistentes com de Fuentes et.al ${ }^{15} \mathrm{em}$ relação aos pré-molares em indivíduos saudáveis. No entanto quando as raízes do presente estudo foram analisadas de acordo com os critérios de Chohayeb et.al ${ }^{14}$, observou-se que os pré-molares inferiores dos portadores de FL/PNS apresentaram 30,2\% dos dentes com angulação $\geq 20^{\circ}$.

Com relação a avaliação dos terços radiculares, verificou-se que $\circ$ apical e o medial foram os que apresentam maior frequência de dilaceração nos portadores de fissuras $(27,7 \%$ e $25,3 \%$ respectivamente). Em indivíduos saudáveis, maior frequência nos terços apical e médio também foram relatados por Malcic et.al ${ }^{13}$. Fuentes et.al ${ }^{15}$, em indivíduos saudáveis observaram que a maioria das curvaturas tinham origem no terço apical $(72,25 \%)$.

Em pesquisa na Tailândia, Wangsrimongkol et.al ${ }^{25}$ avaliaram em indivíduos portadores FLP/PNS a prevalência de anomalias dentarias na maxila e mandíbula. A dilaceração radicular foi definida como uma angulação acentuada da raiz, e através de análise visual encontraram prevalência de 6,4\%. A FLP foi o fenótipo que apresentou maior frequência de dilaceração. Este tipo de fenda também foi - mais afetado pela dilaceração nos trabalhos de Al Jamal et.al ${ }^{23}$ e Raducanu et.al ${ }^{24}$ (19,2\% e $15 \%$ respectivamente). De acordo com a classificação de Chohayeb et.al ${ }^{14}$, a nossa pesquisa revelou preva- 
lências bem mais elevadas de dilaceração nas FL e FP ( $30,9 \%$ e $30,8 \%$ respectivamente). Essa diferença de resultados se deve a ausência de estudos que considerem a dilaceração radicular de acordo com a angulação $\geq 20^{\circ}$.

Weckwerth et.al ${ }^{18}$ analisaram todas as unidades dentárias de indivíduos com FLP/PNS, e consideraram dilaceração quando a angulação radicular foi $\geq 90^{\circ}$. Observaram maior frequência de dilaceração em portadores de FLP $(31,2 \%)$, e em seguida, a FL $(29,6 \%)$. Dentre as unidades dentarias avaliadas, os terceiros molares foram os que apresentaram maior prevalência de dilaceração radicular. Hamasha et.al ${ }^{16}$ que também consideraram a dilaceração como angulação $\geq 90^{\circ} \mathrm{em}$ estudo com indivíduos saudáveis, também constataram maior prevalência desta anomalia nos terceiros molares $(19,2 \%)$. Em nossa pesquisa, os terceiros molares não foram incluídos na amostra, visto que estes dentes anatomicamente já apresentam curvatura acentuada de suas raízes. Ademais, não encontramos em nossos resultados raízes com angulação $\geq 90^{\circ}$.

A análise dos resultados foi completada com o estudo do risco relativo da curvatura radicular $\geq 20^{\circ} \mathrm{em}$ associação aos diferentes tipos de fissura. Observou-se que o risco de dilaceração nos pré-molares esquerdos na presença de FP foi duas vezes maior do que em relação a FLP. Mesmo na ausência de significância estatística, este resultado sugere uma associação preferencial da dilaceração de determinados grupos de dentes em relação ao tipo de fissura. Esse tipo de análise da curvatura radicular ainda não foi encontrado na literatura em portadores de $\mathrm{FL} / \mathrm{PNS}$. O risco relativo de outras anomalias dentárias em fissurados já foram bem documentados, em especial a agenesia, dentro e fora da área da fissura. ${ }^{9,2}$

\section{Conclusão}

Esta pesquisa, realizada através de uma análise mais precisa da curvatura radicular em portadores de FL/PNS, não verificou diferenças no grau de curvatura nos diferentes tipos de fissuras. $O$ risco de dilaceração radicular nos pré-molares esquer- dos na presença de FP foi duas vezes maior do que em relação a FLP. Esses resultados sugerem a realização de estudo caso-controle para que questões relativas às diferenças de angulação entre os portadores de $\mathrm{FL} / \mathrm{PNS}$ e a população em geral, sejam respondidas.

\section{Conflitos de interesses}

Nenhum conflito financeiro, legal ou político envolvendo terceiros (governo, empresas e fundações privadas, etc.) Foi declarado para nenhum aspecto do trabalho submetido (incluindo mas não limitandose a subvenções e financiamentos, conselho consultivo, desenho de estudo, preparação de manuscrito, análise estatística, etc).

\section{Referências}

1. Stanier P, Moore GE. Genetics of cleft lip and palate: syndromic genes contribute to the incidence of non-syndromic clefts. Hum Mol Genet. 2004;13(1): 73-81. doi: 10.1093/ hmg/ddh052

2. Mossey PA, LittleJ, Munger RG, Dixon MJ, Shaw WC. Cleft lip and palate. Lancet. 2009;374(9703):1773-85. doi: $10.1016 / 50140-6736(09) 60695-4$

3. Carinci F, Scapoli L, Palmieri A, Zollino I, Pezzetti F. Human genetic factors in nonsyndromic cleft lip and palate: an update. Int J Pediatr Otorhinolaryngol. 2007;71(10): 15091519. doi: 10.1016/i.ijporl.2007.06.007

4. Harville EW, Wilcox AJ, Lie RT, Abyholm F, Vindenes $H$. Epidemiology of cleft palate alone and cleft palate with accompanying defects. Eur J Epidemiol. 2007;22(6):389-95. doi: $10.1007 / \mathrm{s} 10654-007-9129-y$

5. Meng L, Bian Z, Torensma R, Von den Hoff JW. Biological Mechanisms in palatogenesis and cleft palate. J Dent Res. 2009;88(1):22-23. doi: $10.1177 / 0022034508327868$

6. Spina V, Psillakis JM, Lapa FS, Ferreira MC. Classificação das fissuras lábio palatinas: sugestão de modificação. Rev Hosp Clin Fac Med S Paulo. 1972;27(1):5-6.

7. Letra A, Menezes R, Granjeiro JM, Vieira AR. Defining subphenotypes for oral clefts based on dental development. J Dent Res. 2007;86(10):986-91. doi: $\underline{10.1177 / 154405910708601013}$

8. Paranaíba LMR, Coletta RD, Swerts MSO, Quintino RP, de Barros LM, Martelli-Júnior H. Prevalence of Dental Anomalies in Patients With Nonsyndromic Cleft Lip and/or Palate in a Brazilian Population. Cleft Palate Craniofac J. 2013;50(4):400-5. doi: 10.1597/11-029 
9. Sá J, Araújo L, Guimarães L, Maranhão S, Lopes G, Medrado A et al. Dental anomalies inside the cleft region in individuals with nonsyndromic cleft lip with or without cleft palate. Med Oral Patol Oral Cir Bucal. 2016;21(1):48-52.

10. Shapira Y, Lubit E, Kuftinec MM. Hypodontia in children with various types of clefts. Angle Orthod. 2000;70(1):16-21.

11. Ranta, R. A review of tooth formation in children eith cleft lip/palate. Am J Orthod Dentofacial Orthop. 1986;90(1):1 18.

12. Colak H, Bayraktar Y, Hamidi MM, Tan E, Colak T. Prevalence of root dilacerations in Central Anatolian Turkish dental patients. West Indian Med J. $2012 ; 61$ (6):635-9.

13. Malcic A, Jukic S, Brzovic V, Miletic I, Pelivan I, Anic I. Prevalence of root dilaceration in adult dental patients in Croatia. Oral Surg Oral Med Oral Pathol Oral Radiol Endod. 2006; 102(1):104-9. doi: 10.1016/i.tripleo.2005.08.021

14. Chohayeb AA, Washington DC. Dilaceration of permanent upper lateral incisors: frequency, direction and endodontic treatment implication. Oral Surg. 1983;55(5):519-20.

15. Fuentes R, Arias A, Navarro P, Ottone N, Bucchi C. Morfometría de premolares mandubulares en radiografías panorámicas digitales; análisis de curvaturas radiculares. Int J Morphol. 2015;33(2):476-482. doi: 10.4067/S071795022015000200012

16. Hamasha AA, Al-Khateeb T, Darwazeh A. Prevalence of dilaceration in Jordanian adults. Int Endod J. 2002;35(11):910-912.

17. Miloglu O, Cakici F, Caglayan F, Yilmaz AB, Demirkaya F. The prevalence of root dilacerations in a Turkish population. Med Oral Patol Oral Cir Bucal. 2010;15(3):441-4.

18. Weckwerth GM, Santos CF, Brozoski DT, Centurion BS, Pagin O, Lauris JRP et al. Taurodontism, Root Dilaceration, and Tooth Transposition: A Radiographic Study of a Population With Nonsyndromic Cleft Lip and/or Palate. Cleft Palate Craniofac J. 2016;53(4):404-12. doi: 10.1597/14-299

19. Gorlin RJ, Goldman HM. Thoma's oral pathology. 6th ed. St Louis: Mosby,

1970.

20. da Silva BF, Costa LED, Beltrao RV, Rodrigues TL, de Farias RL, Beltrao RTS. Prevalence asseeement of root dilaceration in permanent incisors. Dental Press J Orthod. 2012 ; 17(6):97-102. doi: 10.1590/\$2176$\underline{94512012000600020}$

21. Regezi JA, Sciubba JJ. Patologia bucal: correlações clínicopatológicas. 3.ed. Rio de Janeiro: Guanabara Koogan. 2000.
22. Nabavizadeh MR, Shamsi MS, Moazami F, Abbaszadegan A. Prevalence of root dilaceration in adult patients referred to Shiraz Dental School (2005-2010). J Dent Shiraz Uni Med Sci. 2013;14(4):160-164.

23. Al Jamal GA, Hazza'a AM, Rawashden MA. Prevalence of dental anomalies in a population of cleft lip anda palate patients. Cleft Palate Craniofacial J. 2010;47(4):413-420. doi: $10.1597 / 08-275.1$

24. Raducanu AM, Didilescu AC, Feraru IV, Dumitrache $M A$, Hantoiu TA, lonescu $E$. Considerations on morphlogical abnormalities of permanent teeth in children with cleft lip and palate. Rom J Morphol Embyol. 2015;56(2):453-457.

25. Wangsrimongkol T, Manosudprasit $M$, Pisek $P$, Chittiwatanapong N. Prevalence and Types of Dental Anomaly in a Thai Non-Syndromic Oral Cleft Sample. J Med Assoc Thai, 2013;96(9):25-35.

26. Sá J, Mariano LC, Canguçu D, Coutinho TSL, Hoshi R, Medrado AP et al. Dental Anomalies in a Brazilian Cleft Population. Cleft Palate Craniofac J. 2016;53(6):714-19. doi: $10.1597 / 14-303$ 